International Journal of Modern Physics B, (C) World Scientific Publishing Company

\title{
THE CUMULANT EXPANSION FOR THE ANDERSON LATTICE WITH FINITE U: THE COMPLETENESS PROBLEM
}

\author{
M. E. FOGLIO* \\ Instituto de Física "Gleb Wataghin", Universidade Estadual de CCampinas, UNICAMP \\ 13083-970 Campinas, São Paulo, Brasil $\mathrm{U}$ \\ and \\ M. S. FIGUEIRA \\ Instituto de Física, Universidade Federal Fluminense, UFF \\ 24210-340 Niterói, Rio de Janeiro, Brasil \\ Received (received date) \\ Revised (revised date)

\begin{abstract}
"Completeness" (i.e. probability conservation) is not usually satisfied in the cumulant expansion of the Anderson lattice when a reduced state space is employed for $U \rightarrow \infty$. To understand this result, the well known "Chain" approximation is first calculated for finite $U$, followed by taking $U \rightarrow \infty$. Completeness is recovered by this procedure, but this result hides a serious inconsistency that causes completeness failure in the reduced space calculation. Completeness is satisfied and the inconsistency is removed by choosing an adequate family of diagrams. The main result of this work is that using a reduced space of relevant states is as good as using the whole space.
\end{abstract}

\section{Introduction}

With the Hubbard operators one can describe complex local states, as well as making substantial simplifications in the study of configurations with a very large number of states by considering only those few states that are relevant to the problem 2 . The cumulant expansion with Hubbard operators has been employed to calculate approximate Green's functions (GF), both for the Hubbard model 1 : and for the Anderson lattice峴, but probability is not conserved in the Anderson lattice when the state with two local electrons at the same site is projected out of the four dimensional space of the local states at each site in the infinite repulsion case. As this problem does not appear in the Hubbard model when the whole space is considered 3 , it is important to find out whether this is an essential consequence of reducing the space. By considering one of the simplest family of diagrams in the cumulant expansion, we can explicitly show that the basic problem is caused by an inconsistency that is already present in the full space calculation. The main

\footnotetext{
*Associate Member of ICTP,Trieste,Italy

†Instituto de Física "Gleb Wataghin", Universidade Estadual de Campinas,UNICAMP, C.P. 6165,13083-970 Campinas, São Paulo, Brazil
} 
conclusion of the present work is that the results obtained by employing a given family of diagrams in the cumulant expansion would not be worse because of the space reduction technique employed in conjunction with the Hubbard operators. Although the space reduction is not essential in the Anderson lattice, our result gives us confidence in the use of that technique when the study of the full space is not viable (e.g. with ions like $E u^{3+}$ that has a ground $f^{7}$ configuration with 3432 states).

The Anderson lattice is an important tool in the study of strongly correlated systems, and there are several reviews devoted to this and closely related problems 6. The model consist of a lattice with two localized electronic states at each site, that are correlated by a strong Coulomb repulsion $U$, plus a band of uncorrelated conduction electrons (c-electrons) that hybridize with the localized electrons (felectrons).

The cumulant expansion has been employed by several authors to study the Ising and the Heisenberg models 0 , 6 , while Hubbard 1 extended the method to a quantum problem with fermions. He applied the cumulant approach to the model of correlated electrons which he had created, and studied with a different technique 10. The method he employed consisted in a perturbative expansion around the atomic limit, using the hopping as perturbation, and introducing the Hubbard operators $X_{j, a b}=|j, b\rangle\langle j, a|$ which transform the local state $|a\rangle$ at site $j$ into the local state $|b\rangle$ at the same site. These X-operators make it possible to describe in a simple way the projection of a system into the subspace of the more relevant states, but they do not satisfy the usual commutation properties of Bose or Fermi operators. Nevertheless, it is possible with the use of cumulants, to derive a diagrammatic expansion involving unrestricted lattice sums of connected diagrams, which satisfies a linked cluster theorem $\mathbf{Z}$. Other expansions with cumulants have been also applied to study fundamental problems in solid state physics 11, 12, 23 , and the relation of some of those treatments to the one presented here will be briefly commented in the final conclusions. The technique employed by Hubbard 14 introduces Grassman external fields without physical meaning (unlike the magnetic fields of the corresponding Ising expansions), but this method seems to be the natural extension of the usual expansion with Fermi operators 0 .

In the present work we shall consider a cumulant expansion of the Anderson lattice, which is an extension 45 of the technique employed by Hubbard to study his model. The hopping of the conduction electrons as well as the intra-site Coulomb repulsion $U$ between the localized (or f-electrons) are included in the unperturbed Hamiltonian, and the hybridization is employed as a perturbation. At a given site $\mathrm{j}$, the state space of the f-electrons is spanned by four states: the vacuum state $|j, 0\rangle$, the two states $|j, \sigma\rangle$ of one f-electron with spin component $\sigma \hbar / 2$ and the state $|j, d\rangle$ with two electrons of opposite spin. In the limit of infinite electronic repulsion $(U \rightarrow \infty)$ the state $|j, d\rangle$ is empty, and one can then consider a reduced space of states by projecting $|j, d\rangle$ out. In this space, the identity $I_{j}$ at site $\mathrm{j}$ should then satisfy the completeness relation: 


$$
X_{j, 00}+X_{j, \sigma \sigma}+X_{j, \overline{\sigma \sigma}}=I_{j} \quad,
$$

where $\bar{\sigma}=-\sigma$, and the three $X_{j, a a}$ are projectors into the three states $|j, 0\rangle$ and $|j, \sigma\rangle$ of the basis. Because of the translational invariance, the statistical averages of $X_{j, a a}$ (denoted by $n_{a}$ ) are independent of $\mathrm{j}$, and from Eq. (1) they should satisfy

$$
n_{0}+n_{\sigma}+n_{\bar{\sigma}}=1
$$

a relation called "completeness" in what follows. It has been found that this relation is not usually satisfied when $|j, d\rangle$ is projected out of the space of local electrons and the $n_{a}$ are calculated with approximate Green's functions (GF) proximation displaying this behavior is the "Chain Approximation" (CHA) 4. 15, 6 , which is the more general cumulant expansion with only second order cumulants. This approximation is $\Phi$-derivable 16 , and has other interesting properties further commented in the Appendix. To better understand the problem, we have studied the system for finite $U$ and then taking $U \rightarrow \infty$, a procedure that will be called $\mathrm{CHU}$ in the present work. As in the case of the Hubbard model in the full spaceB, the probability is conserved in the Anderson lattice by this procedure. The analysis of the method shows that to secure the completeness, the CHU has to employ different values for the probabilities of the two states $|j, \sigma\rangle$ and $|j, \bar{\sigma}\rangle$, which should be equal in the paramagnetic state, and this inconsistency is what causes the lack of completeness found with the CHA in the reduced space. This is the basic result of the present work, that shows that the calculation in the full space satisfies completeness through an important inconsistency, that is hidden in the formalism.

A systematic way of adding a set of diagrams to an arbitrary family, so that completeness be satisfied by the $n_{a}$ calculated with the corresponding GF, has been proposed and verified in a number of cases 16 . When applied to the CHA, this conjecture gives the "Complete Chain Approximation" (CCHA) which satisfies Eq. (2) but it is not $\Phi$-derivable any more. It seems interesting to repeat the calculation for the diagrams of the CCHA employing a finite $U$ and keeping $|j, d\rangle$ in the space of local states, and finally taking $U \rightarrow \infty$ : this procedure shall be called CCHU in what follows. One finds that completeness is also satisfied by the CCHU, and that the occupation numbers of the two states $|j, \sigma\rangle$ and $|j, \bar{\sigma}\rangle$ are equal, and coincide with the value calculated directly for the CCHA. This last result, as well as the details of the calculation, show that the procedure of making simplified models by

projecting unoccupied states out of the space of local states, can give adequate results.

\section{The Anderson Lattice with Finite $U$}

The Anderson lattice with finite $U$ is described by the Hamiltonian

$$
H=\sum_{\mathbf{k}, \sigma} E_{\mathbf{k}, \sigma} C_{\mathbf{k}, \sigma}^{\dagger} C_{\mathbf{k}, \sigma}+\sum_{j \sigma} E_{j, \sigma} f_{j \sigma}^{\dagger} f_{j \sigma}+\sum_{j} U f_{j \sigma}^{\dagger} f_{j \sigma} f_{j \bar{\sigma}}^{\dagger} f_{j \bar{\sigma}}+
$$


The completeness in cumulant expansion

$$
\sum_{j k \sigma}\left(V(k) \exp \left(i \mathbf{k} . \mathbf{R}_{\mathbf{j}}\right) f_{j \sigma}^{\dagger} C_{k \sigma}+H . C .\right)
$$

where $C_{k \sigma}^{\dagger}\left(C_{k \sigma}\right)$ is the usual creation (destruction) operator of conduction band electrons with wavevector $\mathbf{k}$ and spin component $\sigma \hbar / 2$, and the $f_{j \sigma}^{\dagger}\left(f_{j \sigma}\right)$ are those corresponding to the local (f) electrons at site $j$. To make the connection with the Hubbard operators one should substitute

$$
f_{j \sigma}=X_{j, 0 \sigma}+\sigma X_{j, \bar{\sigma} d}
$$

into Eq. (3), where the factor $\sigma= \pm 1$ is necessary to obtain the correct phase of the states. As the present treatment employs the Grand Canonical Ensemble of electrons it is convenient to introduce

$$
\mathcal{H}=H-\mu\left\{\sum_{\mathbf{k}, \sigma} C_{\mathbf{k}, \sigma}^{\dagger} C_{\mathbf{k}, \sigma}+\sum_{j a} \nu_{a} X_{j, a a}\right\} \quad,
$$

where $X_{j, a a}$ is the occupation number operator of state $\mid a>$ at site $j$, and $\nu_{a}$ is the number of electrons in that state. The exact and unperturbed averages of the operator $A$ are respectively denoted by $\langle A\rangle_{\mathcal{H}}$ and $\langle A\rangle$, and it is also convenient to introduce

$$
\varepsilon_{j, a}=E_{j, a}-\mu \nu_{a}
$$

and

$$
\varepsilon_{\mathbf{k}_{\sigma}}=E_{\mathbf{k}_{\sigma}}-\mu
$$

because the energies $E_{j, a}$ of all ionic states $\mid a>$ and the energies $E_{\mathbf{k}, \sigma}$ of the conduction electrons appear only in that form in all the calculations.

Operators $X_{j, a b}$ do not satisfy the usual anticommutation (commutation) properties of the Fermi (Bose) operators when the two operators are at the same site, but in this case it is sufficient to employ the product rules

$$
X_{j, a b} X_{j, c d}=\delta_{b, c} X_{j, a d}
$$

One has more freedom to define the operation between pairs of operators when they are at different sites, and it is convenient to choose properties as close as possible to those of the usual Fermi or Bose operators. It is then convenient to say that $X_{j, a b}$ is of the "Fermi type" ("Bose type") when the number of electrons in the two states $|j, a\rangle$ and $|j, b\rangle$ differ by an odd (even) number. The best choice is then to use anticommutation relations when the two operators at different sites are of the "Fermi type" and commutation relations otherwise.

The Anderson lattice has been studied in the case of $U \rightarrow \infty$ resorting to a cumulant expansion $\mathbf{6}$ that employs the last term in $H$ (hybridization) as a perturbation, and this treatment is an extension of the expansion already employed by Hubbard for his model 1,10 . The Hubbard operators $X_{j, a b}$ were used in this limit to 
eliminate the doubly occupied states $|j, d\rangle$ from the state space of the f-electrons at site $j$. The projection of $H$ into the reduced space is

$$
\begin{aligned}
H_{r}= & \sum_{\mathbf{k}, \sigma} E_{\mathbf{k}, \sigma} C_{\mathbf{k}, \sigma}^{\dagger} C_{\mathbf{k}, \sigma}+\sum_{j, \sigma} E_{j, \sigma} X_{j, \sigma \sigma} \\
& +\sum_{j, \mathbf{k}, \sigma}\left(V_{j, \mathbf{k}, \sigma} X_{j, 0 \sigma}^{\dagger} C_{\mathbf{k}, \sigma}+V_{j, \mathbf{k}, \sigma}^{*} C_{\mathbf{k}, \sigma}^{\dagger} X_{j, 0 \sigma}\right)
\end{aligned}
$$

The method has been generalized to several configurations with a rather arbitrary choice of states 5 , and can be used to study the Anderson lattice with finite $U$, described by the Hamiltonian in Eq. (3). The cumulant expansion gives the GF of the Matsubara type for imaginary time $\tau$

$$
\left\langle\left(\widehat{X}_{j, \alpha}(\tau) \widehat{X}_{j^{\prime}, \alpha^{\prime}}\right)_{+}\right\rangle_{\mathcal{H}}
$$

where $\widehat{X}_{j, \alpha}(\tau)=\exp (\tau \mathcal{H}) X_{j, \alpha} \exp (-\tau \mathcal{H})$ corresponds to the Heisenberg representation, the subindex $\alpha=(b, a)$ represents the transition $|a>\rightarrow| b>$ and the subindex + indicates that the operators inside the parenthesis are taken in the order of increasing $\tau$ to the left, with a change of sign when the two Fermi-type operators have to be exchanged to obtain this ordering.

The GFs in Eq. (10) are defined in the interval $0 \leq \tau \leq \beta \equiv 1 / T$ and, because of their boundary condition in this variable 5 , one can associate to them a Fourier series with a coefficient for each Matsubara frequency $\omega_{\nu}=\pi \nu / \beta$ (where $\nu$ are the even or odd integers, depending on whether the operator $X_{\alpha}$ is of the Bose type or of the Fermi type respectively). One can also transform the GF to reciprocal space 0 and, because of the invariance against time and lattice translations, they are proportional to quantities $G_{\alpha \alpha^{\prime}}^{f f}\left(\mathbf{k}, \omega_{\nu}\right)$. One can also associate these quantities to the $i \omega_{\nu}$ points of a complex plane in the variable $z=\omega+i y$ and, in the usual way 17, one can make the analytic continuation to the upper and lower half-planes of the complex frequency $z$, obtaining a function $\bar{G}_{\alpha \alpha^{\prime}}^{f f}(\mathbf{k}, \mathbf{z})$ which is minus the Fourier transform of the real time GF. By an adequate choice of $\alpha$ and $\alpha^{\prime}$ it is possible to use these functions to calculate the occupation $n_{a}$ of the local state $|a\rangle$ :

$$
n_{a}=\int_{-\infty}^{\infty} \rho_{b a}(\omega) f_{T}(\omega) d \omega
$$

where

$$
f_{T}(z)=\{1+\exp (\beta z)\}^{-1}
$$

is the Fermi function and

$$
\rho_{b a}(\omega)=\frac{1}{\pi} \lim _{\epsilon \rightarrow 0} \operatorname{Im}\left\{\frac{1}{N_{s}} \sum_{\mathbf{k}} \bar{G}_{b a, a b}^{f f}(\mathbf{k}, \omega+\mathbf{i}|\epsilon|)\right\}
$$


is the spectral density associated to the transition $\alpha=(b, a)$. With the same $\rho_{b a}(\omega)$ it is also possible to obtain the occupation $n_{b}$ of the local state $|b\rangle$ :

$$
n_{b}=\int_{-\infty}^{\infty} \rho_{b a}(\omega)\left(1-f_{T}(\omega)\right) d \omega
$$

When the state $\mid d>$ is projected out, the occupation $n_{\sigma}$ can be calculated with only Eq. (11) and $\bar{G}_{0 \sigma, \sigma 0}^{f f}$, while in the whole space one could also use Eq. (14) and $\bar{G}_{\sigma d, d \sigma}^{f f}$. These two values of $n_{\sigma}$ are not automatically equal when one uses approximate GFs, and this property is the origin of the results obtained in the present work.

\subsection{The chain approximation (CHA)}

The family of diagrams which give this approximation is shown in figure 1. In the diagrams of the cumulant expansion $\mathbf{0}$, the circles (vertices) correspond to cumulants: the filled ones for the f-electrons and the empty ones for the c-electrons. In the present expansion the hybridization is the perturbation, and it is represented in the diagrams by the lines (edges) joining two vertices. Because of the form of the hybridization in Eq. (3), the edges can only join a c-vertex to an f-vertex. The number of edges reaching a vertex gives the order of the cumulant, and only those of second order appear in the CHA. All the cumulants of second order coincide with the free propagators, and because of Wick's theorem, these are the only nonzero cumulants of the c-electrons, while there are cumulants of any even order for the f-electrons, which can be calculated employing a generalized version of Wick's theorem 16 . 18

\subsubsection{The Anderson lattice for $U \rightarrow \infty$ in the reduced space}

The f-electron GF has been calculated for the CHA 9 in the reduced space, and using the simpler notation $G_{0 \sigma, \sigma 0}(\mathbf{k}, \mathbf{z}) \equiv \overline{\mathbf{G}}_{\mathbf{0} \sigma, \sigma}^{\mathbf{f f}} \mathbf{0}(\mathbf{k}, \mathbf{z})$ we can write

$$
G_{0 \sigma, 0 \sigma}(\mathbf{k}, \mathbf{z})=-\frac{\mathbf{D}_{\mathbf{0}_{\sigma}}^{\mathbf{0}}\left(\mathbf{z}-\varepsilon_{\mathbf{k}_{\sigma}}\right)}{\left(\mathbf{z}-\varepsilon_{\mathbf{1}}(\mathbf{k})\right)\left(\mathbf{z}-\varepsilon_{\mathbf{2}}(\mathbf{k})\right)} \quad .
$$

The energies $\varepsilon_{1}(\mathbf{k})$ and $\varepsilon_{2}(\mathbf{k})$ are those of the two elementary excitations with wave vector $\mathbf{k}$, resulting from the hybridization of a band $\varepsilon_{\mathbf{k} \sigma}$ and a dispersionless band of energy $\varepsilon_{f}=\varepsilon_{j \sigma}$ with a reduced hybridization constant $\sqrt{D_{0 \sigma}^{0}} V(k)$. They are given by the two roots of $\left(z-\varepsilon_{f}\right)\left(z-\varepsilon_{\mathbf{k} \sigma}\right)-D_{0 \sigma}^{0}|V(k)|^{2}=0$, where

$$
D_{0 \sigma}^{0}=\left\langle X_{00}+X_{\sigma \sigma}\right\rangle
$$

and $\langle A\rangle$ is the unperturbed average of $A$. This GF is equal to that of two uncorrelated bands of energies $\varepsilon_{f}$ and $\varepsilon_{\mathbf{k} \sigma}$ hybridized with a parameter $\sqrt{D_{0 \sigma}^{0}} V(k)$, 
showing that the only effect of the correlations in the CHA is to reduce the hybridization by a factor $\sqrt{D_{0 \sigma}^{0}}$. A typical spectral density $\rho_{0 \sigma}(\omega)$ is shown in figure 2, and it is easy to show that

$$
n_{0}+n_{\sigma}=\int_{-\infty}^{\infty} d \omega \rho_{0 \sigma}(\omega)=D_{0 \sigma}^{0} \quad,
$$

i.e. $n_{0}+n_{\sigma}$, which is the total area of the spectral density in the CHA, coincides with the unperturbed value of the same quantity.

\subsubsection{The Anderson lattice for $U \rightarrow \infty$ in the full space}

For the Anderson lattice with finite $U$, described by the Hamiltonian in Eq. (3), the same family of diagrams shown in figure 1 corresponds to the CHA. One can employ the generalized method introduced in ref. $\mathrm{G}$ for a rather arbitrary choice of states, and obtain the approximate GFs for finite $U$. In reciprocal space and for the complex frequency $z$ they are

$$
\begin{gathered}
G_{0 \sigma, 0 \sigma}(k, z)=-\frac{D_{0 \sigma}^{0}(1-b)}{\left(z-\varepsilon_{f}\right)(1-a-b)}, \\
G_{\bar{\sigma} d, 0 \sigma}(k, z)=-\frac{D_{\bar{\sigma} d}^{0} \sigma a}{\left(z-\varepsilon_{f}-U\right)(1-a-b)}, \\
G_{0 \sigma, \bar{\sigma} d}(k, z)=-\frac{D_{0 \sigma}^{0} \sigma b}{\left(z-\varepsilon_{f}\right)(1-a-b)}, \\
G_{\bar{\sigma} d, \bar{\sigma} d}(k, z)=-\frac{D_{\bar{\sigma} d}^{0}(1-a)}{\left(z-\varepsilon_{f}-U\right)(1-a-b)},
\end{gathered}
$$

where

$$
\begin{gathered}
a=\frac{D_{0 \sigma}^{0}|V(k)|^{2}}{\left(z-\varepsilon_{f}\right)\left(z-\varepsilon_{\mathbf{k}_{\sigma}}\right)}, \\
b=\frac{D_{\bar{\sigma} d}^{0}|V(k)|^{2}}{\left(z-\varepsilon_{f}-U\right)\left(z-\varepsilon_{\mathbf{k}_{\sigma}}\right)}, \\
D_{\bar{\sigma} d}^{0}=\left\langle X_{\overline{\sigma \sigma}}+X_{d d}\right\rangle,
\end{gathered}
$$

and $D_{0 \sigma}^{0}$ is given in Eq. (16). However, it must be noted that now we are employing $<A>$ to indicate the unperturbed average of $A$ in the full space, i.e. for the Hamiltonian of Eq. (3) taken with $V(k)=0$.

The identity in the full space is given by

$$
X_{j, 00}+X_{j, \sigma \sigma}+X_{j, \overline{\sigma \sigma}}+X_{j, d d}=I_{j}
$$

rather than by Eq. (1), and the completeness with finite $U$ then becomes

$$
n_{0}+n_{\sigma}+n_{\bar{\sigma}}+n_{d}=1
$$


Note that completeness is automatically satisfied for the unperturbed case, because then $n_{0}+n_{\sigma}+n_{\bar{\sigma}}+n_{d}=D_{0 \sigma}^{0}+D_{\bar{\sigma} d}^{0}=1$, and it is worth analyzing the two limit cases $U=0$ and $U \rightarrow \infty$ for the chain approximation.

The $U=0$ case Employing

$$
<<f_{j \sigma} ; f_{j \sigma}^{\dagger}>>=G_{0 \sigma, 0 \sigma}+\sigma G_{\bar{\sigma} d, 0 \sigma}+\sigma G_{0 \sigma, \bar{\sigma} d}+G_{\bar{\sigma} d, \bar{\sigma} d}
$$

we obtain the exact GF in this limit, and completeness is satisfied. Although this result is not surprising, it serves to illustrate the nature of the $X$ operators, which contain strong correlations as a result of their own definition. This is clearly shown by employing Eqs. (19,20) to calculate $\left\langle X_{j, 0 \sigma} X_{j, \bar{\sigma} d}\right\rangle$ and $\left\langle X_{j, \bar{\sigma} d} X_{j, 0 \sigma}\right\rangle$ : these averages should be zero because of the rules of product of the $X$ operators (i.e. Eq. (8)), but a different value is obtained in the CHA. This result can be interpreted as follows: Wick's theorem is satisfied by the Fermion operators $f_{j \sigma}$ and $f_{j \sigma}^{\dagger}$, and all the cumulants of order higher than two are zero, so that the CHA gives the exact solution for these operators. As Wick's theorem is not satisfied by the $X$ operators, all the diagrams with cumulants higher than two are missing from the individual GF in Eqs. (18 -21), and they are not exact in the CHA. It is by a cancellation automatically built in the formalism that Eq. (27) gives the exact $<<f_{j \sigma} ; f_{j \sigma}^{\dagger}>>$, although the $G_{\alpha \alpha^{\prime}}^{f f}$ are only approximate.

The relation $X_{j, 0 \sigma} X_{j, \bar{\sigma} d}=0$ is an example of the "kinematic restriction which forbids more than one energy level of a single ion be occupied" ed. The failure of that restriction to be satisfied in the average by many approximate GF of the Heisenberg and related models is discussed in 20. A cure of this problem for the Heisenberg model with uniaxial anisotropy is proposed in that reference, and we shall later return to this property in connection with our problem.

The $U \rightarrow \infty$ case To study this limit one should start with a very large $U$. In the usual case, the band of conduction electrons is close to $\varepsilon_{f}$, and from Eq. (23) it is $b \rightarrow 0$ when $U \rightarrow \infty$. A fairly symmetrical situation is obtained when the conduction band is close to $\varepsilon_{f}+U$, and in this case it follows that $a \rightarrow 0$ when $U \rightarrow \infty$. When both $\varepsilon_{f}$ and $\varepsilon_{f}+U$ are far from the conduction band and $U \rightarrow \infty$, both $b \rightarrow 0$ and $a \rightarrow 0$, and the GF coincide with those of the unperturbed problem. In all cases the two "cross" GFs, i.e. $G_{\bar{\sigma} d, 0 \sigma}$ and $G_{0 \sigma, \bar{\sigma} d}$ of Eqs. (19, 20), vanish for apy value of $z$ when $U \rightarrow \infty$, so that the kinematic restriction discussed in reference 20 is automatically satisfied in this limit of the CHA.

Let us consider only the case when $\varepsilon_{f}$ is close to the conduction electron band. The Eq. (18) then becomes

$$
G_{0 \sigma, 0 \sigma}(\mathbf{k}, \mathbf{z})=-\frac{\mathbf{D}_{\mathbf{0}_{\sigma}}^{\mathbf{0}}\left(\mathbf{z}-\varepsilon_{\mathbf{k}_{\sigma}}\right)}{\left(\mathbf{z}-\varepsilon_{\mathbf{1}}(\mathbf{k})\right)\left(\mathbf{z}-\varepsilon_{\mathbf{2}}(\mathbf{k})\right)}
$$


which is just the same Eq. (15) obtained in the CHA when the state $|j, d\rangle$ with two $\mathrm{f}$ electrons is projected out. In the two ways of dealing with $|j, d\rangle$, Eq. (17) shows that $n_{0}+n_{\sigma}$ coincides with the unperturbed value $D_{0 \sigma}$, and the effect of the perturbation consist in transferring weights between $n_{0}$ and $n_{\sigma}$. Equation (21) becomes

$$
G_{\bar{\sigma} d, \bar{\sigma} d}(\mathbf{k}, \mathbf{z})=-\frac{\mathbf{D}_{\bar{\sigma} \mathbf{d}}^{\mathbf{0}}}{\left(\mathbf{z}-\varepsilon_{\mathbf{f}}-\mathbf{U}\right)},
$$

and the corresponding $\rho_{\bar{\sigma} d}$ is a delta function with weight $D_{\bar{\sigma} d}^{0}$ at $\varepsilon_{f}+U$. Applying Eq. (11) and Eq. (14) to $\rho_{\bar{\sigma} d}$ we obtain $n_{d}=D_{\bar{\sigma} d}^{0} f_{T}\left(\varepsilon_{f}+U\right)$ and $n_{\bar{\sigma}}=D_{\bar{\sigma} d}^{0}(1-$ $\left.f_{T}\left(\varepsilon_{f}+U\right)\right)$. The relation $n_{\bar{\sigma}}+n_{d}=D_{\bar{\sigma} d}^{0}$ is then valid independently of the value of $\mu$, and together with Eq. (17) shows that the completeness in the full space is satisfied: $n_{0}+n_{\sigma}+n_{\bar{\sigma}}+n_{d}=D_{0 \sigma}^{0}+D_{\bar{\sigma} d}^{0}=1$. Notice that when $\varepsilon_{f}$ is close to the conduction electron band, $\varepsilon_{f}+U$ is very large and $n_{d}=D_{\bar{\sigma} d}^{0} f_{T}\left(\varepsilon_{f}+U\right) \rightarrow 0$, so that completeness in the reduced space $n_{0}+n_{\sigma}+n_{\bar{\sigma}}=1$ is also satisfied. Also the unperturbed $n_{d}^{0}=0$, so that from $n_{\bar{\sigma}}=D_{\bar{\sigma} d}^{0}$ follows that $n_{\bar{\sigma}}=n_{\bar{\sigma}}^{0}$, and this value is usually different from the $n_{\sigma}$ calculated by the CHA. This shows that completeness is satisfied in the present calculation at the cost of using different values for $n_{\sigma}$ and $n_{\bar{\sigma}}$. As Eqs. (28,29) are valid for the two values of $\sigma$, different values of $n_{\sigma}$ are obtained when they are calculated with $G_{0 \sigma, 0 \sigma}$ vs. $G_{\sigma d, \sigma d}$. When the state $|j, d\rangle$ is projected out, the Eq. (29) cannot be used, and one is then forced to calculate $n_{\bar{\sigma}}$ with $G_{0 \bar{\sigma}, 0 \bar{\sigma}}$, so that $n_{\bar{\sigma}}=n_{\sigma}$, and completeness can not be satisfied by the CHA in the reduced space. The different values of $n_{\bar{\sigma}}$ and $n_{\sigma}$ obtained with the CHU are shown in figures 3 and 4 as a function of $T$ for the following parameters: $E_{j, \sigma}=-0.5$, a local hybridization with $V=0.3$ and a rectangular conduction band centered at $E_{\mathbf{k}, \sigma}=0$ and with a total width $2 W=\pi$. The chemical potential in the two figures is respectively $\mu=-0.60$ and $\mu=-0.40$, and the corresponding dotted curves show the crossover, that occurs at low $T$ in the system with $V=0$, from the maximal occupation $n_{\sigma}^{0}=0.5$ of the local electron to the empty state $n_{\sigma}^{0}=0$.

In many works 3421 it is the $\mathrm{GF}<<f_{j \sigma} ; f_{j \sigma}^{\dagger}>>$ rather than the partial GF that is calculated, and completeness is satisfied in the full space. As it was shown above, this could hide the fact that there is a pair of conflicting values of $n_{\sigma}$ for each $\sigma$, and that different values of $n_{\bar{\sigma}}$ and $n_{\sigma}$ are necessary in the paramagnetic case to satisfy completeness: the relation Eq.(26) would not be satisfied if only one of the two possible values were used for both $n_{\bar{\sigma}}$ and $n_{\sigma}$. The correct completeness obtained from $<<f_{j \sigma} ; f_{j \sigma}^{\dagger}>>$ masks this basic failure of the CHA, and the properties calculated in the $\mathrm{CHU}$ would not be more reliable than those obtained in the CHA when $|j, d\rangle$ is projected out.

\section{The Complete Chain Approximation (CCHA)}

A conjecture that gives a systematic way of achieving completeness by adding a set of diagrams to an arbitrary family was stated in a previous paper 16 , and it was verified in a number of cases that include diagrams with infinite fourth-order 
cumulants, but a general derivation has not been found. This conjecture gives for the CHA the "Complete Chain Approximation" (CCHA) which satisfies Eq. (2), and the extra diagrams are shown in figure 5 . We shall now apply to this family of diagrams the procedure (named CCHU in this case) employed in Section , of keeping $|j, d\rangle$ in the space of local states for finite $U$ and then taking $U \rightarrow \infty$. As completeness in the full space is satisfied by the CHU, one wonders whether adding the extra diagrams to this approximation would maintain Eq. (26). This equation is satisfied in the $\mathrm{CHU}$ at the cost of having different occupation numbers for the two states $|j, \sigma\rangle$ and $|j, \bar{\sigma}\rangle$, and one also wonders whether this inconsistency would be removed in the CCHU. In this section it is shown that the two questions have an affirmative answer, and that the corresponding $n_{\sigma}$ and $n_{\bar{\sigma}}$ coincide with the value calculated directly in the CCHA.

The fact that different average values of the same operator can be obtained from different GF that_correspond to the same approximation has been well known in magnetic models 20 , and in that reference they propose that when a given correlation function is calculated, the diagrams should be classified according to the number of momentum variables summed over in the diagram and be consistentlyincluded in the calculation according to this classification (see also references 2223). This procedure is closely related to the conjecture stated in 16 , but this last gives clear rules for the diagrams that should be included in a GF for the_Anderson lattice as well as for other systems of fermions. The procedure stated in 20 associates a given family of diagrams to the calculation of a given average rather than to a GF: when their prescription is applied to our problem, we have a GF in the CHA without momentum integrations, but it is necessary to integrate once over the wave vectors when the number of electrons is calculated, so that further diagrams should be included. Our conjecture seems more clear because it applies directly to the GF, and has the same effect as the proposal in 20 .

\subsection{The GF in the CCHU}

The GFs obtained for finite $U$ in the CCHA are rather complicated, and only the corrections $\Delta G_{\alpha, \alpha^{\prime}}(\mathbf{k}, \mathbf{z})$ that could give a non-zero contribution to the occupation numbers when $U \rightarrow \infty$ are given below. The calculation of these quantities follows

the same technique employed in the Appendix $\mathrm{C}$ of reference 16 to derive the function $S_{\sigma}^{C H}\left(\mathbf{k}, \omega_{\nu}\right)$, which corresponds to the present $\Delta G_{0 \sigma, 0 \sigma}\left(\mathbf{k}, \omega_{\nu}\right)$ when $U \rightarrow \infty$. We obtain:

$$
\begin{aligned}
\Delta G_{0 \sigma, 0 \sigma}\left(\mathbf{k}, \omega_{\nu}\right)= & -|V(k)|^{2}\left\{A K_{0 \sigma}\left(\omega_{\nu}\right)+B K_{0 \sigma}^{2}\left(\omega_{\nu}\right) C_{\sigma}\left(\omega_{\nu}\right)+\right. \\
& \left.B^{\prime} K_{0 \sigma}^{2}\left(\omega_{\nu}\right) H_{0 d}\left(\omega_{\nu}\right)+C K_{0 \sigma}^{2}\left(\omega_{\nu}\right)\right\} \\
\Delta G_{\bar{\sigma} d, \bar{\sigma} d}\left(\mathbf{k}, \omega_{\nu}\right)= & -|V(k)|^{2}\left\{-A K_{\bar{\sigma} d}\left(\omega_{\nu}\right)+B K_{\bar{\sigma} d}^{2}\left(\omega_{\nu}\right) C_{\sigma}\left(\omega_{\nu}\right)+\right. \\
& \left.B^{\prime} K_{\bar{\sigma} d}^{2}\left(\omega_{\nu}\right) H_{0 d}\left(\omega_{\nu}\right)-C K_{\bar{\sigma} d}^{2}\left(\omega_{\nu}\right)\right\}
\end{aligned}
$$


and we do not give the expressions for $G_{\bar{\sigma} d, 0 \sigma}(k, z)$ and $G_{0 \sigma, \bar{\sigma} d}(k, z)$ because, as in the CHA, they do not contribute to $n_{a}$ when $U \rightarrow \infty$. The following definitions have been used:

$$
\begin{gathered}
K_{a b}\left(\omega_{\nu}\right)=-\frac{1}{i \omega_{\nu}+\left(\varepsilon_{a}-\varepsilon_{b}\right)} \\
C_{\sigma}\left(\omega_{\nu}\right)=\frac{1}{N} \sum_{\mathbf{k}} \frac{\left(i \omega_{\nu}-\varepsilon_{f}\right)}{\left(i \omega_{\nu}-\varepsilon_{1}\right)\left(i \omega_{\nu}-\varepsilon_{2}\right)} \\
\beta H_{0 d}\left(\omega_{\nu}\right)=\sum_{\omega_{1}} C_{\sigma}\left(\omega_{1}\right) K_{\overline{0} d}\left(\omega_{\nu}+\omega_{1}\right)
\end{gathered}
$$

and both $\varepsilon_{1}=\varepsilon_{1}(\mathbf{k})$ and $\varepsilon_{2}=\varepsilon_{2}(\mathbf{k})$ have been given just after Eq. (15). We have left out from the constants in Eqs. (30,31) all those terms that would vanish when $U \rightarrow \infty$ (note in particular that $\left\langle X_{d d}\right\rangle=0$ ): they can then be expressed by $A=\left\langle X_{00}\right\rangle\left\langle X_{\sigma \sigma}\right\rangle \beta I_{1}-D_{0 \sigma}^{0} I_{2} ; B=-\left\langle X_{\sigma \sigma}\right\rangle\left(1+D_{0 \sigma}^{0}\right) ; B^{\prime}=D_{0 d}^{0}=-\left\langle X_{00}\right\rangle$ and $C=-D_{0 \sigma}^{0} I_{1}$, where

$$
I_{\ell}=\frac{1}{\beta} \sum_{\omega_{1}} C_{\sigma}\left(\omega_{1}\right) K_{\sigma}^{\ell}\left(\omega_{1}\right) \quad(\text { with } \quad \ell=1,2) \quad .
$$

These values of $A, B$ and $C$ are equat to the parameters with the same name in Eq. (4.5) of reference 16, while the parameter $B^{\prime}$ only appears in the present treatment. The terms with $A, B$ and $C$ in Eqs. (30.31) are of the same type of the corresponding ones in reference 16 , and the usual analytic continuation from their values at all the points $\varsigma_{\nu}=i \omega_{\nu}$ to the complex plane $\varsigma$ give analytic functions off the real axis.

The sum over $\omega_{1}$ in Eq. (34) was obtained by the usual technique, and the resulting $H_{0 d}\left(\omega_{\nu}\right)$ has several terms in which $f_{T}\left(\varepsilon_{d}-i \omega_{\nu}\right)$ is present. To obtain an analytic function off the real axis by analytic continuation of the values of $H_{0 d}\left(\omega_{\nu}\right)$, it is necessary to use the following property of $f_{T}(z)$ :

$$
f_{T}\left(\varepsilon_{d}-i \omega_{\nu}\right)=\left(1-\exp \left(\beta \varepsilon_{d}\right)\right)^{-1} \equiv-b_{T}\left(\varepsilon_{d}\right)
$$

where $b_{T}(z)$ is the Bose equivalent to the $f_{T}(z)$. It then follows that

$$
\begin{aligned}
H_{0 d}\left(\omega_{\nu}\right)= & \frac{f_{T}\left(\varepsilon_{1}\right)-f_{T}\left(\varepsilon_{2}\right)}{\varepsilon_{1}-\varepsilon_{2}} \\
& -\frac{i \omega_{\nu}-\varepsilon_{d}+\varepsilon_{f}}{\varepsilon_{1}-\varepsilon_{2}}\left\{\frac{f_{T}\left(\varepsilon_{1}\right)+b_{T}\left(\varepsilon_{d}\right)}{i \omega_{\nu}-\varepsilon_{d}+\varepsilon_{1}}-\frac{f_{T}\left(\varepsilon_{2}\right)+b_{T}\left(\varepsilon_{d}\right)}{i \omega_{\nu}-\varepsilon_{d}+\varepsilon_{2}}\right\} \\
& +b_{T}\left(\varepsilon_{d}\right) \frac{i \omega_{\nu}-\varepsilon_{d}+\varepsilon_{f}}{\left(i \omega_{\nu}-\varepsilon_{d}+\varepsilon_{1}\right)\left(i \omega_{\nu}-\varepsilon_{d}+\varepsilon_{2}\right)}
\end{aligned}
$$

\footnotetext{
${ }_{\ddagger}$ But note that there is a typing mistake in that reference: the expression for $B$ should read $B=-\left(D_{\sigma}^{0}\left(1-D_{\sigma}^{0}\right)+x_{\sigma}\right)$.
} 
and the analytic continuation of the total GF would also be an analytic function off the real axis, making it possible to employ Eqs. (11.14) to calculate the occupation numbers.

\subsection{The occupation numbers for the Anderson lattice in the CCHU}

It is possible to obtain partial results that are fairly independent of the system parameters by integrating Eqs. (11,14) over $\omega$ for each value of $\mathbf{k}$, and leaving the sum over this variable as a final step. One calculates the occupation number $n_{a}(\mathbf{k})$ associated to the wavevector $\mathbf{k}$ and the state $|a\rangle$ by substituting the local spectral density $\rho_{b a}(\omega)$ in Eq. (11) or Eq. (14) by

$$
\rho_{b a}(\mathbf{k}, \omega)=\frac{\mathbf{1}}{\pi} \lim _{\epsilon \rightarrow \mathbf{0}} \operatorname{Im}\left\{\overline{\mathbf{G}}_{\mathbf{b a}, \mathbf{a b}}^{\mathbf{f f}}(\mathbf{k}, \omega+\mathbf{i}|\epsilon|)\right\} .
$$

Defining $D_{0 \sigma}(\mathbf{k})=\mathbf{n}_{\mathbf{0}}(\mathbf{k})+\mathbf{n}_{\sigma}(\mathbf{k})$ and $D_{\bar{\sigma} d}(\mathbf{k})=\mathbf{n}_{\bar{\sigma}}(\mathbf{k})+\mathbf{n}_{\mathbf{d}}(\mathbf{k})$ we obtain that

$$
D_{0 \sigma}(\mathbf{k})=\mathbf{D}_{\mathbf{0}_{\sigma}}^{\mathbf{0}}-|\mathbf{V}(\mathbf{k})|^{\mathbf{2}} \mathbf{A}
$$

and

$$
D_{\bar{\sigma} d}(\mathbf{k})=\mathbf{D}_{\bar{\sigma} \mathbf{d}}^{\mathbf{0}}+|\mathbf{V}(\mathbf{k})|^{\mathbf{2}} \mathbf{A} \quad,
$$

so that

$$
n_{0}(\mathbf{k})+\mathbf{n}_{\sigma}(\mathbf{k})+\mathbf{n}_{\bar{\sigma}}(\mathbf{k})+\mathbf{n}_{\mathbf{d}}(\mathbf{k})=\mathbf{D}_{\mathbf{0} \sigma}^{\mathbf{0}}+\mathbf{D}_{\bar{\sigma} \mathbf{d}}^{\mathbf{0}}=\mathbf{1} \quad .
$$

The sum of Eq. (41) over all the $N$ values of $\mathbf{k}$ divided by $N$ shows that completeness (i.e. Eq.(26)) is satisfied by any set of system parameters in the CCHU, in particular by any unperturbed density $\rho_{c}^{0}(\varepsilon)$ of c-electron states with respect to their energy and by any $V(k)$.

Using Eqs. (28,30) we find that

$$
\begin{aligned}
n_{\sigma}(\mathbf{k})= & D_{0 \sigma}^{0}\left\{\frac{\varepsilon_{1} f_{T}\left(\varepsilon_{1}\right)-\varepsilon_{2} f_{T}\left(\varepsilon_{2}\right)}{\varepsilon_{1}-\varepsilon_{2}}-\varepsilon_{\mathbf{k}_{\sigma}} \frac{f_{T}\left(\varepsilon_{1}\right)-f_{T}\left(\varepsilon_{2}\right)}{\varepsilon_{1}-\varepsilon_{2}}\right\} \\
& -|V(k)|^{2}\left\{A f_{T}\left(\varepsilon_{f}\right)+B g\left(\varepsilon_{\mathbf{k} \sigma}\right)+C \beta f_{T}\left(\varepsilon_{f}\right)\left(1-f_{T}\left(\varepsilon_{f}\right)\right)\right\}
\end{aligned}
$$

where

$$
g\left(\varepsilon_{\mathbf{k} \sigma}\right)=\frac{f_{T}\left(\varepsilon_{f}\right)}{\left(\varepsilon_{f}-\varepsilon_{1}\right)\left(\varepsilon_{f}-\varepsilon_{2}\right)}+\frac{f_{T}\left(\varepsilon_{1}\right)}{\left(\varepsilon_{1}-\varepsilon_{f}\right)\left(\varepsilon_{1}-\varepsilon_{2}\right)}+\frac{f_{T}\left(\varepsilon_{2}\right)}{\left(\varepsilon_{2}-\varepsilon_{f}\right)\left(\varepsilon_{2}-\varepsilon_{1}\right)},
$$

and employing Eq. (39) one obtains

$$
n_{0}(\mathbf{k})=\mathbf{D}_{\mathbf{0}_{\sigma}}-\mathbf{n}_{\sigma}(\mathbf{k}) \quad .
$$

From Eqs. (29,31) follows that $n_{d}(\mathbf{k})=\mathbf{0}$, and therefore

$$
n_{\bar{\sigma}}(\mathbf{k})=\mathbf{D}_{\bar{\sigma} \mathbf{d}}(\mathbf{k})=\mathbf{D}_{\bar{\sigma} \mathbf{d}}^{\mathbf{0}}+|\mathbf{V}(\mathbf{k})|^{\mathbf{2}} \mathbf{A} \quad .
$$


From these expressions is not obvious a priori that one would obtain $n_{\bar{\sigma}}=n_{\sigma}$ after summing the Eqs. (42,45) over $\mathbf{k}$, and this property was tested by numerical calculation with a rectangular $\rho_{c}^{0}(\varepsilon)$. The agreement was perfect, indicating that one should be able to prove analytically this property, but we have not made any attempt in such direction. A different approach is to compare Eqs. (39, 42) with the corresponding expressions that were obtained in the calculation of the CCHA 16: it was verified that the expressions for $n_{\sigma}(\mathbf{k})$ and for $D_{0 \sigma}(\mathbf{k})$ are identical and therefore $n_{0}, n_{\sigma}$ and $D_{0 \sigma}=n_{0}+n_{\sigma}$ are also equal in the two methods. Since there is not an independent expression for $n_{\bar{\sigma}}$ in the CCHA, the paramagnetic condition $n_{\bar{\sigma}}=n_{\sigma}$ was imposed 16 and completeness then reads as

$$
D_{0 \sigma}+n_{\sigma}=1 \quad,
$$

which was shown 16 to be satisfied for several $\rho_{c}^{0}(\varepsilon)$ and $V(k)$. Since $D_{0 \sigma}$ and $n_{\sigma}$ are identical in the two methods, this relation is then satisfied in the CCHU when is true in the CCHA.

Equation (41) shows that when $U \rightarrow \infty$, completeness (viz. Eq. (26)) is satisfied for any $\rho_{c}^{0}(\varepsilon)$ and $V(k)$ in the full space, and employing $n_{d}(\mathbf{k})=\mathbf{0}$ (and therefore $\left.n_{d}=0\right)$ there follows that

$$
D_{0 \sigma}+n_{\bar{\sigma}}=1 \quad .
$$

Taking the difference of Eq. (46) and Eq. (47), it follows that $n_{\bar{\sigma}}=n_{\sigma}$ in the $\mathrm{CCHU}$ and that these values coincide with those calculated with the CCHA, at least for all the $\rho_{c}^{0}(\varepsilon)$ and $V(k)$ for which Eq. (2) was numerically verified for this approximation. This result shows that employing the conjecture proposed in reference 16 , the results obtained by the $\mathrm{CCHA}$ in the reduced space, that had the state $|j, d\rangle$ projected out, are compatible with those calculated by the CCHU in the full space. The occupation number of the two spin states is unique in the CCHU, thus removing the difficulty presented by the CHA.

The inconsistency that is brought to light by the use of the CHU is the difference between the values of $n_{\bar{\sigma}}$ and $n_{\sigma}$ obtained in that calculation. The dashed curves in figures 3 and 1 plot the value of $n_{\sigma}$ calculated with the $\mathrm{CHU}$, which we have shown to be equal to the $n_{\sigma}$ obtained in the CHA, and it is set to be equal to the $n_{\bar{\sigma}}$ in this last approximation because that quantity can not be independently obtained in the reduced space. The calculation in the full space makes possible to obtain an independent value of $n_{\bar{\sigma}}$ in the $\mathrm{CHU}$, which as discussed before is equal to the unperturbed $n_{\sigma}^{0}$, that is shown in figures 3 and 1 by the dotted curves. The different occupation of $n_{\bar{\sigma}}$ and $n_{\sigma}$ in the CHU is clearly shown at low $T$ in the two figures, and these are the values that have to be employed to satisfy completeness (viz. Eq. (26) in the full space and Eq. (2) when $U \rightarrow \infty$ ). It is then clear that it is not possible to satisfy Eq. (2) in this region when the relation $n_{\bar{\sigma}}=n_{\sigma}$ is forced by the CHA. The $n_{\sigma}$ in the CCHU coincides with the $n_{\sigma}$ in the CCHA, and it is given by the full line. 
It is interesting to remark, that the terms with $A$ and $C$ in Eqs. (30 and 31) are proportional to $\left\langle X_{00}\right\rangle\left\langle X_{\sigma \sigma}\right\rangle / T=n_{\sigma}^{0} n_{0}^{0} / T$. As this contribution is proportional to $1 / T$, it could be dominant at very low $T$ when $0.1<\varepsilon_{f} / T<10$, but it is very small outside this interval because either $\left\langle X_{00}\right\rangle$ or $\left\langle X_{\sigma \sigma}\right\rangle$ is then much smaller than $T$. The region inside this interval is the intermediate valence region, and corresponds, at low $T$, to a crossover from the Kondo region to a region of a thermally excited local moment. All the results are fairly well behaved when $\varepsilon_{f}$ is not too small. The whole contribution of Eq. (30) is equal to the difference between the dashed curve $\left(n_{\sigma}\right.$ for the CHU) and the full curve ( $n_{\sigma}$ for the CCHU). This contribution is proportional to $n_{\sigma}^{0}$, and the two curves coincide for very small $T$ in figure 3 while they tend to different values in figure 1 because in this case the $n_{\sigma}^{0}$ does not vanish for $T \rightarrow 0$.

The value of $n_{\sigma}$ as a function of $\mu$ for $T=0.1$ is plotted in figure 6 for the same approximations used in the previous figures, showing a monotonic increase with $\mu$ as it could be expected.

\section{Concluding Remarks}

The Anderson lattice is a model that gives a schematic description of many real systems with strongly correlated electrons, and it seems important to understand better the different methods employed to calculate its properties. In this work we consider the cumulant expansion, a perturbative method that has many desirable properties, and we employ the Hubbard operators, that make it easy to project the space of the system into a subspace of states of interest. The main motivation of using the Hubbard operators is that they are very well suited to reduce the space of states of the system by eliminating those that are no relevant to the properties considered.

There are several perturbative expansions that use this operators, and here we shall briefly compare three of them: 4,2224 . All these expansions employ the Matsubara technique 25 with imaginary times, and first expand the grand partition function or the Green's functions as a series of statistical averages of time ordered products of operators that contain successive orders of the interaction Hamiltonian. The technique developed by Keiter, Kimball and Grewe and reviewed in reference 24, expresses these averages in terms of a diagramatic expansion that leads to a scheme of the Brillouin-Wigner type 26.27. This method has been already compared with our treatment in more detail $\mathrm{E}$, and shall not be further discussed here.

We shall consider now the method in 12 , and show that it employs a type of cumulants different from the ones used in our treatment.The initial averages are reduced in 22 by a technique already used by Gaudin 28 to prove the standard Wick 's theorem, and later applied to averages of spin operators18 and to Hubbard operators of the Fermi type 16. This technique takes advantage of the fact that the commutator of two Hubbard operators is a linear combination of the same type of operators, making it possible to reduce an average with $n$ Hubbard operators into a linear combination of averages with only $n-1$ of them. In 12 they proceed with 
this reduction until only averages of diagonal Hubbard operators (that are therefore of the Bose type) remain, and these averages are then expressed in terms of cumulants that can be obtained from an explicitly given generating function. A diagramatic expansion that employs these cumulants at single sites is then obtained, and a renormalization of vertices can be also performed 13,21 .

Our expansion, on the other hand, closely follows the original derivation employed for the Ising modelt,8,29, and the initial averages are expressed directly in terms of cumulants, employing a general property that was extended to include cumulants with Hubbard operators of the Fermi type( cf. Theorem 3.1 in reference 5 ). A diagramatic expansion different from that in 12 is then obtained, and the same reduction method 16 is employed to calculate the expressions of the cumulants.

Although the two methods are related, the one described in 12 is different from ours, and their cumulants correspond to different operators. The cumulants in 12 only contain "diagonal operators" that are of the Bose type, while the Hubbard operators that appear in the cumulants of our expansion are of the Fermi type. In particular, the cumulants that are of order $n$ in 12 are of order $2 n$ in our treatment. As discussed in the Appendix for the CHA, the relations that are related by Englert to the classical Ward identities take in our case a rather different physical meaning than the one corresponding to the Ising problem, because in that problem the momentum operators are linear combination of Hubbard operators of the Bose type, while they are of the Fermi type in our case.

One difficulty in the cumulant expansion we employ, is that the approximate GF obtained do not usually satisfy completeness (i.e. conservation of probability). In the present work we consider the cumulant expansion of the Anderson lattice, and employ the Hubbard operators to project, in the limit of $U \rightarrow \infty$, the unoccupied state with two electrons out of the space of local electrons. It has been shown 16 that the "chain approximation" (CHA), which corresponds to the most general family of diagrams with only second order cumulants, does not satisfy completeness in the reduced space. It was useful to analyze the problem by keeping the doubly occupied state for finite $U$ within this approximation, and then taking $U \rightarrow \infty$. It was shown that completeness is satisfied when this procedure is used, but to obtain this result it is necessary to use different values of $n_{\sigma}$ and $n_{\bar{\sigma}}$, that are obtained respectively employing Eq. (11) and Eq. (14) with the two GFs $G_{0 \sigma, 0 \sigma}$ and $G_{\bar{\sigma} d, \bar{\sigma} d}$ associated with the creation or destruction of the same spin $\sigma$ (cf. Eq. (27)). To exemplify this situation employing either of figures 3 or 4, note that to satisfy completeness it is necessary to use the dotted curve for one spin and the dashed curve for the opposite spin. When the GF is calculated in the reduced space (without the state $|d\rangle)$ there is no $G_{\bar{\sigma} d, \bar{\sigma} d}$, and the paramagnetic condition $n_{\sigma}=n_{\bar{\sigma}}$ has to be forced on the system. The dashed line has then to be used for the occupation of both spin components, and completeness is therefore not satisfied when the dashed curve is different from the dotted one. One important consequence of our derivation is that although the calculations employing the full space do satisfy completeness, they might hide the use of inconsistent values of $n_{\sigma}$ and $n_{\bar{\sigma}}$. 
A conjecture on how to obtain families of diagrams that satisfy completeness has been proposed 16, and it was verified for some families of diagrams that might contain any number of fourth order cumulants 30 . The "complete chain approximation" satisfies completeness and was obtained by adding diagrams to the CHA according to the proposed conjecture. It seemed then relevant to analyze the completeness problem in the CCHA by considering again the full space for finite $U$ and then taking $U \rightarrow \infty$. It was found that the $n_{\sigma}$ and $n_{\bar{\sigma}}$ obtained when employing the two GFs $G_{0 \sigma, 0 \sigma}$ and $G_{\bar{\sigma} d, \bar{\sigma} d}$ satisfy $n_{\sigma}=n_{\bar{\sigma}}$ and are also equal to the $n_{\sigma}$ that was calculated with the CCHA, which only employs $G_{0 \sigma, 0 \sigma}$. We conclude that the inconsistency pointed out for the CHA disappears for the CCHA. Moreover, we could find the occupation number $n_{a}(\mathbf{k})$ associated to the wavevector $\mathbf{k}$ and the state $|a\rangle$ for $a=0, \sigma, \bar{\sigma}$,(cf. Section ) because it is possible to calculate those values analytically. Employing $G_{0 \sigma, 0 \sigma}$ we have also found that the $n_{\sigma}(\mathbf{k})$ and $n_{0}(\mathbf{k})$, calculated in CCHU are identical to those calculated employing the CCHA.

The interest in the use of the Hubbard operators is that given a model with local states, it is a fairly simple procedure to reduce the corresponding space of states to a subspace that only contains the few states that are considered more relevant; e.g.: to study the Jahn-Teller effect of $\mathrm{Co}^{2+}$ in $\mathrm{MgO}$, the 120 states of the $(3 d)^{7}$ ground configuration of $\mathrm{Co}^{2+}$ have been projected into the 12 ground states that are split by the Coulomb interaction and by the crystal fields 2.31 . The question immediately arises as to what contributions are lost by the use of this method. The technique employed in the CCHU, of comparing the results in the reduced space with those obtained by calculating the properties in the full space and then making the energy of the states that would be removed go to infinity, seems a good test. Although our conclusions strictly apply only to the problem considered, the results summarized in the previous paragraphs show that one can safely use the reduced space when the occupation of the removed states goes to zero.

\section{Acknowledgements}

The authors are grateful to Prof. Roberto Luzzi for critical comments. They would like to acknowledge financial support from the following agencies: CAPES-PICD (MSF), FAPESP and CNPq (MEF). This work was done (in part) in the frame of Associate Membership Programme of the International Centre for Theoretical Physics, Trieste ITALY (MEF).

\section{References}

[1] J. Hubbard, Proc. R. Soc. London, Ser. A 296, 82 (1966).

[2] M. E. Foglio, Brazilian Journal of Physics accepted for publication 8, (1997)

[3] L. Craco and M. A. Gusmão, Phys. Rev. B 52, 17135 (1995).

[4] A. C. Hewson, J. Phys. C: Solid State Phys. 10, 4973 (1977).

[5] M. S. Figueira, M. E. Foglio and G. G. Martinez, Phys. Rev. B 50, 17933 (1994).

[6] P.A. Lee, T.M. Rice, J.W. Serene, L. J. Sham and J. W. Wilkins, Comments 
Cond. Mat. Phys. 12, 99 (1986).

P. Fulde, Solid State Physics. 41, 1 (1988).

P. Schlottmann, Phys.Rep. 181, 1 (1989).

A. C. Hewson, The Kondo problem to Heavy Fermions (Cambridge U.P. Cambridge 1993).

A. Georges, G. Kotliar, W. Krauth and M. J. Rozenberg. Rev. Mod. Phys. 68. 13 (1996).

[7] M. Wortis, in Phase Transitions and Critical Phenomena, edited by C. Domb and M. S. Green (Academic, London, 1974), Vol. 3. pg. 113.

[8] F. Englert, Phys. Rev. 129, 567 (1963).

[9] R. B. Stinchcombe, G. Horwitz, F. Englert and R. Brout, Phys. Rev. 130, 155 (1963).

[10] J. Hubbard, Proc. R. Soc. London, Ser. A 276, 238 (1964); ibid. A 277, 237 (1964); ibid. A 281, 401 (1964).(These are the first three papers of a series of six).

[11] P. Fulde, Electron correlations in Molecules and Solids (Springer Verlag. 1995).

K. W. Becker and P. Fulde, Z. Phys. B. 72, 423 (1988).

K. W. Becker and P. Fulde, J. Chem. Phys. 91, 4223 (1988).

K. W. Becker and W. Brenig, Z. Phys. B. 79, 195 (1990).

[12] Yu. A. Izyumov, B. M. Leffulov, E. V. Shipitsyn, M. Bartkowiak and K. A. Chao, Phys. Rev. B 46, 15697 (1992).

[13] M. Bartkowiak and K. A. Chao, Phys. Rev. B 47, 1616 (1993).

[14] W. Metzner, Phys. Rev. B 43, 8549 (1991).

[15] E. V. Anda, J. Phys. C: Solid State Phys. 14, L1037 (1981).

[16] M. S. Figueira and M. E. Foglio, J. Phys.: Condens. Matter 8, 5017 (1996).

[17] J. W. Negele and H. Orland, Quantum Many-Particle Systems (Addison-Wesley, New York, 1988), Chap. 2.

[18] D. H. Y. Yang and Y. L. Wang, Phys. Rev. B 10, 47141974.

[19] M. S. Figueira and M. E. Foglio, Physica A 208, 279 (1994).

[20] D. H. Y. Yang and Y. L. Wang, Phys. Rev. B 12, 10571975.

[21] M. Bartkowiak and K. A. Chao, Phys. Rev. B 47, 4193 (1993).

[22] R. B. Stinchcombe, J. Phys. C: Solid State Phys. 3, 2266 (1970).

[23] M. G. Cottam and R. B. Stinchcombe, J. Phys. C: Solid State Phys. 3, 2283 (1970).

[24] H. Keiter and G. Morandi Phys. Rep. 109, 227 (1981).

[25] A. A. Abrikosov, L. P. Gorkov and I. E. Szyaloshinski, Methods of Quantum Field Theory in Statistical Physics (Dover, 1975).

[26] N. Grewe and H. Keiter, Phys. Rev. B 24, 4420 (1981).

[27] T. V. Ramakrishnan and K. Sur, Phys. Rev. B 26, 1798 (1982).

[28] M. Gaudin, J. Nucl. Phys. 15, 89 (1960).

[29] C. Bloch and J. S. Langer, J. Math. Phys. 6, 554 (1965).

[30] M. S. Figueira, Ph.D. thesis, Universidade Estadual de Campinas, SP, Brazil, 1994.

[31] L. T. Peixoto and M. E. Foglio, Phys. Rev. B 32, 2596 (1985)

[32] M. Bartkowiak - "High density expansion for narrow band systems" - Ph. D. Thesis - Adam Mickiewicz University - Poznan, Poland, 1988.

[33] G. Baym, Phys. Rev. 127, 1391 (1962).

[34] L. Craco and M. A. Gusmão, Phys. Rev. B 54, 1629 (1996).

\section{Appendix A}


Properties of the Chain Approximation

Although the main purpose of the present paper is not to discuss the CHA, and much less to propose it for the calculation of all the Anderson lattice properties, this approximation has some interesting characteristics that seem worth while presenting in a rather summary way. Within the context of the cumulant expansion of the Anderson lattice model, the CHA was studied by Hewsont and its was recently analyzed in more detail5. 16 .

From the point of view of the high density $1 / z$ classification of diagrams $6,22,22,23$ the CHA is of zeroth order. An interesting feature is that the CHA is a $\Phi$-derivable approximation 16 , generated by the same type of skeleton that gives the molecular field approximation in the Ising and in the quantum Heisenberg model th. Although the present treatment of the Anderson lattice closely follows the cumulant expansion for the Ising model 8 , there is an essential difference with the treatment employed for the magnetic systems, namely that the external fields we employ to generate the cumulants 4.14 require the introduction of the Grassman fields $\xi$, that have no physical meaning and should be set to zero at the end of the calculation. The operators associated to those fields are not magnetic moments, which do not change the number of electrons, but they create or destroy an electron, so that their averages have to be zero when all the $\xi=0$. As a consequence, one does not obtains a self consistent relation equivalent to the Weiss molecular field equation, and for the same reason many of the relations established 1.29 for the Ising model change its meaning in the present context. Nevertheless, the CHA retains some of the characteristics of a mean field approximation 1 : the correlation between the electrons, that in the limit $U=\infty$ would forbid the occupancy of a local electron with spin $\sigma$ at any given site that already has a local electron with spin $-\sigma$, is replaced by an average reduction of the hybridization constant $V^{2}$ by a factor $D_{\sigma}=1-n_{\bar{\sigma}}$, equal for all the sites.

When the general formalism of the diagramatic expansion is replaced by an approximate evaluation, many of the general properties might be lost in the procedure, and one could make a list of properties that would be desirable to maintain 32 . It seems then interesting to analyze the CHA from that perspective, and in particular by considering that it is a $\Phi$ - derivable approximation.

The study of the $\Phi$-derivable approximation 29.33 in the cumulant expansions for the Ising model has peen reviewed by Wortis, and the corresponding treatment for the Hubbard mode 14 and for the Anderson lattice 16 have been also recently discussed, so we shall use the concepts and notations presented in those references to avoid repetition. The basic idea is to replace the many unrenormalized diagrams of the cumulant expansion by fewer skeleton diagrams with renormalized vertices $M_{n}$, and employ a functional $\Phi$ of the $M_{n}$ that corresponds to the complete family of skeletons, to obtain the grand canonical potential $\Omega=T \ln \mathcal{Z}$, where the $\mathcal{Z}$ is the grand partition function. In the $\Phi$-derivable approximation only a subfamily of all the possible skeletons is considered, and three relations that are valid in the exact expansion are employed to define completely the approximate method 7 . These 
three conditions relate the $\ln \mathcal{Z}, M_{n}$ and the "self-fields" $S_{n}$, 14 , and correspond to the Eqs.(3.1,3.2,3.5) given in reference 6 for the exact expansion. One would then expect that as the correlation functions in a $\Phi$-derivable approximation are obtained from "the same underlying free energy", they "will have singular behavior at the same points and obey certain self-consistency conditions" 6 .

Variational properties for the free energy that are valid for the Ising model 829 and for the Heisenberg model22, can be easily extended to the $\Phi$-derivable approximations of the Anderson lattice by making the appropriate changes to the notation 16. These properties guarantee the relation (cf. Eq. (A.28) in $\mathrm{E}$ and Eq. (3.7) in 16$)$

$$
\frac{\delta \ln [\mathcal{Z}(\beta, \xi)]}{\delta \xi(\ell)} \equiv\langle\widehat{Y}(\ell)\rangle_{c}^{V, \xi}=M_{1}(\ell ; \xi)
$$

which corresponds to the Eq. (4.17) in $\mathrm{B}$. Although this relation equates the magnetization to the renormalized cumulant $M_{1}$ in the Ising model, it only gives the trivial identity $0=0$ when $\xi=0$ in the present cumulant expansion. Nevertheless, for $\xi \neq 0$ one can employ

$$
\frac{\delta^{2} \ln [\mathcal{Z}(\beta, \xi)]}{\delta \xi(\ell) \delta \xi\left(\ell^{\prime}\right)} \equiv\left\langle\widehat{Y}(\ell) \widehat{Y}\left(\ell^{\prime}\right)\right\rangle_{c}^{V, \xi}=\frac{\delta M_{1}(\ell ; \xi)}{\delta \xi\left(\ell^{\prime}\right)}
$$

and, following the Appendix A in reference 16, obtain the Eq. (15) employed in the present work in the CHA for $\xi=0$, or the Eqs. 11821) for the CHU. One could also calculate $\left\langle\widehat{Y}(\ell) \widehat{Y}\left(\ell^{\prime}\right)\right\rangle_{c}^{V, \xi}$ directly from the diagramatic expansion, and the two results should be equal; Englert has shown that in the Ising model this equality requires a relation between the vertex and the bond renormalization ( the Eq.(5.22) in reference 1 ), which is very similar to the "Ward identities" of quantum electrodynamics. These identities have been also discussed by Stinchcombe22 for the Heisenberg model. It is a simple matter to show that both the CHA and CHU satisfy the Ward identity in the form stated by Englert, because the only non zero self-fields in the vertex renormalization are the $S_{1}$, and the only diagrams that contribute to the GF $\left\langle\widehat{Y}(\ell) \widehat{Y}\left(\ell^{\prime}\right)\right\rangle_{c}^{V, \xi=0}$ are the simple chains in figure 1, so that the renormalized bond is the same as the bare bond. Another way to prove this relation is to notice that the GF in the diagramatic calculation 1 are equal to those obtained from the $\Phi$-derivable approximation employing the simple skeleton of Figure $3 \mathrm{a}$ in reference 16 , both for the CHA and for the CHU.

Another interesting aspect of the CHA is the calculation of the $\ln \mathcal{Z}$, that would make possible to derive all the thermodynamic properties of the system. By the very simplicity of the skeleton that gives the CHA and the CHU, the only non zero self-fields in this approximation are the $S_{1} 16$, and these are also zero in the physical region when all $\xi=0$, so that $\ln \mathcal{Z}=\ln \mathcal{Z}_{0}$, i.e. we obtain the same grand partition function $\mathcal{Z}_{0}$ of the unperturbed system. All the thermodynamic relations are then automatically satisfied, but at the price of not giving any new information. To obtain a better $\ln \mathcal{Z}$ one can use the GF in the CHA, and the coupling constant 
integration method has been employed to this purpose in reference 19. Another possible method is to integrate

$$
\left(\frac{\partial F}{\partial N_{t}}\right)_{T, V}=\mu \quad,
$$

where $N_{t}$ is the total number of electrons in the system. After solving a puzzling paradox that appears in a parameter region where three solutions are possible for a given $N_{t}$, one can compare the free energies $F$ obtained by these two methods of calculation. In the reference mentioned, their values are plotted for the atomic limit and compared with the available exact solution, showing that the two methods give fairly close values of $F$ that compare reasonably well with the exact curve.

Another very important property of the approximation is the spectral density of the one-electron GF, which is plotted in Figure 2 for the $f$ electrons, in the CHA. This approximation gives the gross behaviour of the spectral density, but it misses the presence of the Kondo peak, that should appear close to the Fermi surface at low $T$ when the system parameters are in the Kondo region. This peak is what gives the characteristically large susceptibility and specific heat of the heavy fermions at low $T$, and its absence from the $\mathrm{CHA}$ and $\mathrm{CHU}$ is to be expected because in this approximation there are no spin flips, that are essential for the existence of the Kondo effect.

To improve on the molecular field approximation one should add the ringd, that are the next order diagrams in the $1 / z$ expansion. A family with infinite fourth order cumulants has been considered, and the corresponding spectral density shows some differences with that of the CHA, but it does not show a Kondo peak either, as can be seen in the figure 5 of reference $\mathrm{B}$. The same problem is therefore present in both the CCHA and the CCHU. One should also mention that employing a similar expansion, the magnetization of the Hubbard model has been calculated by including all the cumulants 13 , but neglecting the spin fluctuations in their calculation: this type of technique would not be then able to recover the Kondo peak in the Anderson lattice. By a similar approximation the exact solution of the Falicov Kimball model has been obtained34, and the spin fluctuations of the Hubbard model have been approximately included in the same work.

The absence of the Kondo peak from the approximate GF that have employed is still under study, and we have been able to find an approximation closely related to the cumulant expansion that shows a structure near the Fermi surface that decreases in intensity when $T$ increases 2 , and could therefore be identified as the Kondo peak.

The present summary shows that the CHA and CHU have very special properties within the cumulant expansion, and the absence of completeness in the CHA seemed particularly intriguing, thus giving the motivation for the present work, that serves to clarify this behavior.

The CCHA is not $\Phi$-derivable, but is satisfies completeness and carries within its structure the elimination of some inconsistencies of the CHA that are only revealed through the study of the CCHU. It seems clear that many valuable approx- 
imations would not satisfy all the properties of the exact solution that one would like to maintain, but we believe that they are nevertheless worth its study and use, provided that their shortcomings are kept in mind. 
Figure 1: The diagrams for the GF in the chain approximation (CHA). The circles (vertices) correspond to cumulants: the filled ones for the f-electrons and the empty ones for the c-electrons. The perturbation (hybridization) is represented in the diagrams by the lines (edges) joining two vertices. a) The CHA diagrams for the f-electrons GF, represented by the filled square to the right. b) Same as a) for the c-electrons, represented by an empty square.

Figure 2: Plot of the spectral density of f-electrons for the CHA as a function of $\omega$ for a rectangular band of total width $2 W=\pi$ centered at $E=0$, with single local electron energy $E_{j, \sigma}=-0.5$ equal to the chemical potential $\mu$, and with hybridization parameter $V=0.3$, all measured in the same energy units. A gap appears at $\mu \approx \varepsilon_{f}=E_{j, \sigma}-\mu$.

Figure 3: The occupation number $n_{\sigma}$ is plotted as a function of $T$ for several different approximations and for the same parameters used in figure 2, but with chemical potential $\mu=-0.60$. The dashed line is $n_{\sigma}$ calculated with the CHU, and coincides with the same parameter calculated with the CHA. The dotted line is $n_{\bar{\sigma}}$ calculated with the $\mathrm{CHU}$, which coincides with the unperturbed value $n_{\sigma}^{0}$ (i.e. for $V=0$ ). The full line is $n_{\sigma}$ calculated with the CCHU and coincides with $n_{\bar{\sigma}}$ calculated within the same approximation and also with the $n_{\sigma}$ obtained with the CCHA. Note that $n_{\bar{\sigma}}$ and $n_{\sigma}$ are different for the CHU (dotted vs. dashed line).

Figure 4: Same as in figure 3 but for $\mu=-0.40$.

Figure 5: a) The Complete Chain Approximation: empty squares symbolize the diagrams of the c-electron GF in the CHA (cf. figure $1 \mathrm{~b}$ b). b) The diagrams added to the CHA to make it complete.

Figure 6: The occupation number $n_{\sigma}$ is plotted as a function of $\mu$ for $T=0.1$ while all the remaining parameters are the same used in figure 2. All the curves are monotonically increasing functions of $\mu$. 
a) $\leftarrow \circ<+<-0-0<+\cdots=\leftarrow<$

b) $\leftarrow$ < $+\leftarrow 0-0<+\cdots=\leftarrow \square<$ 


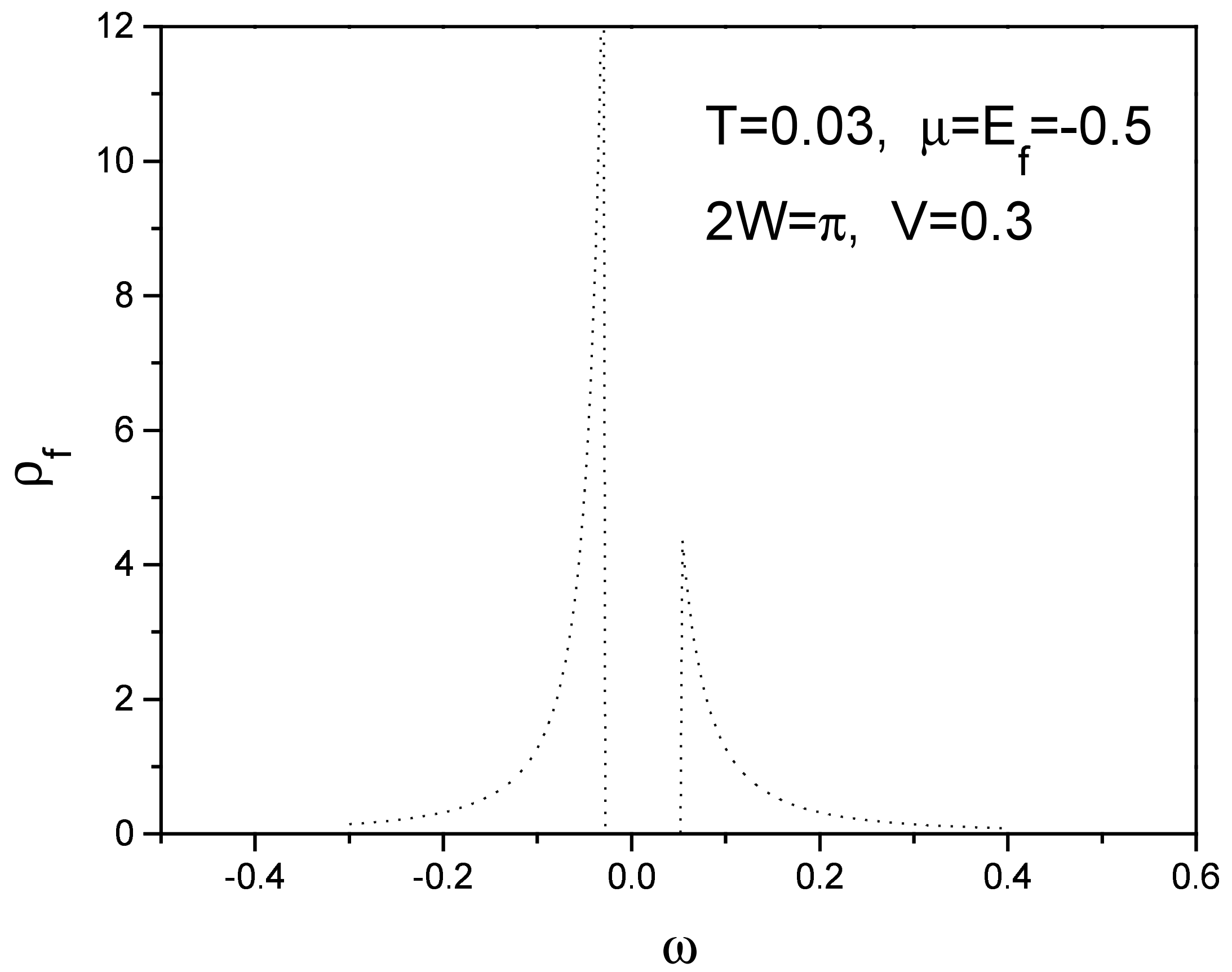




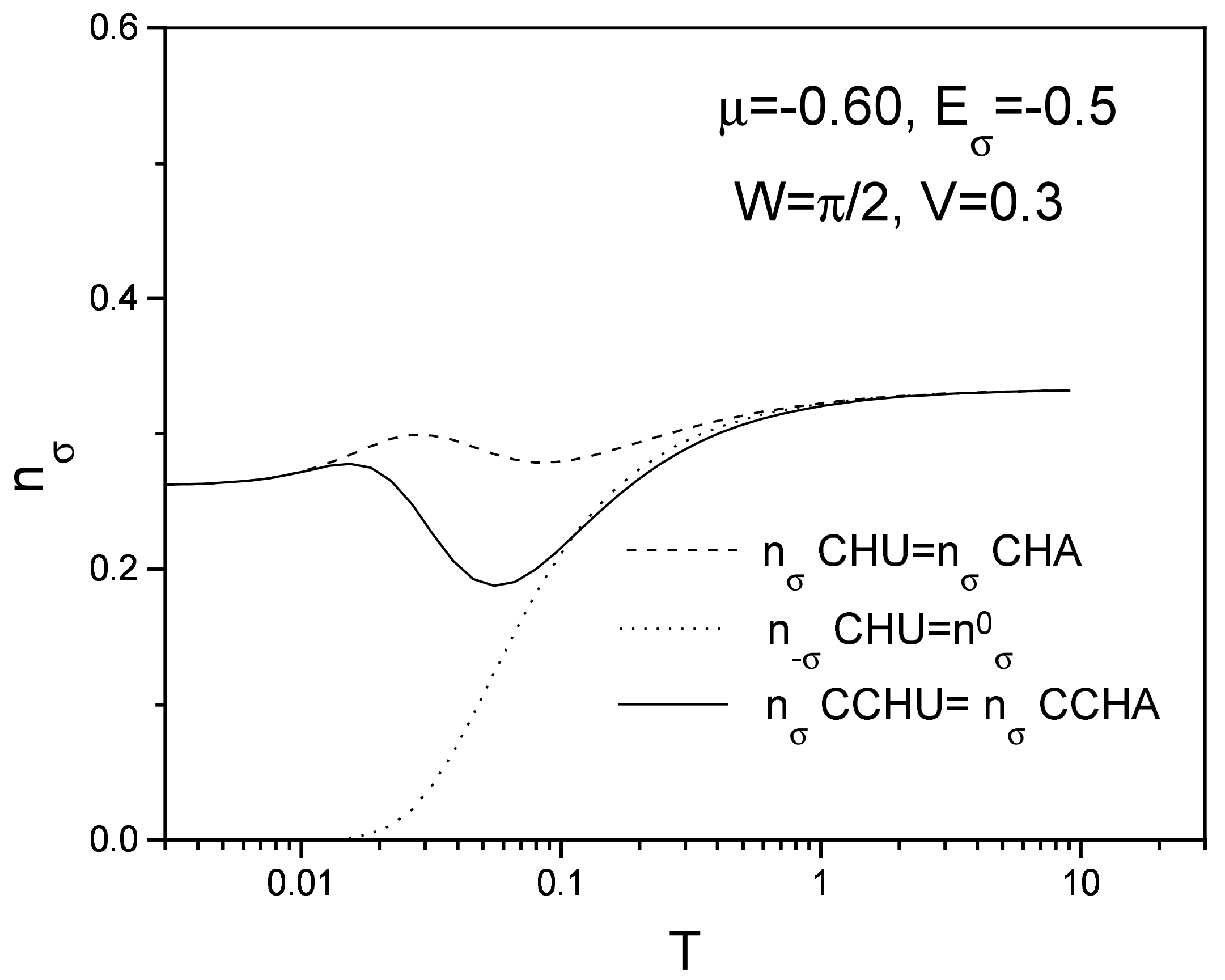




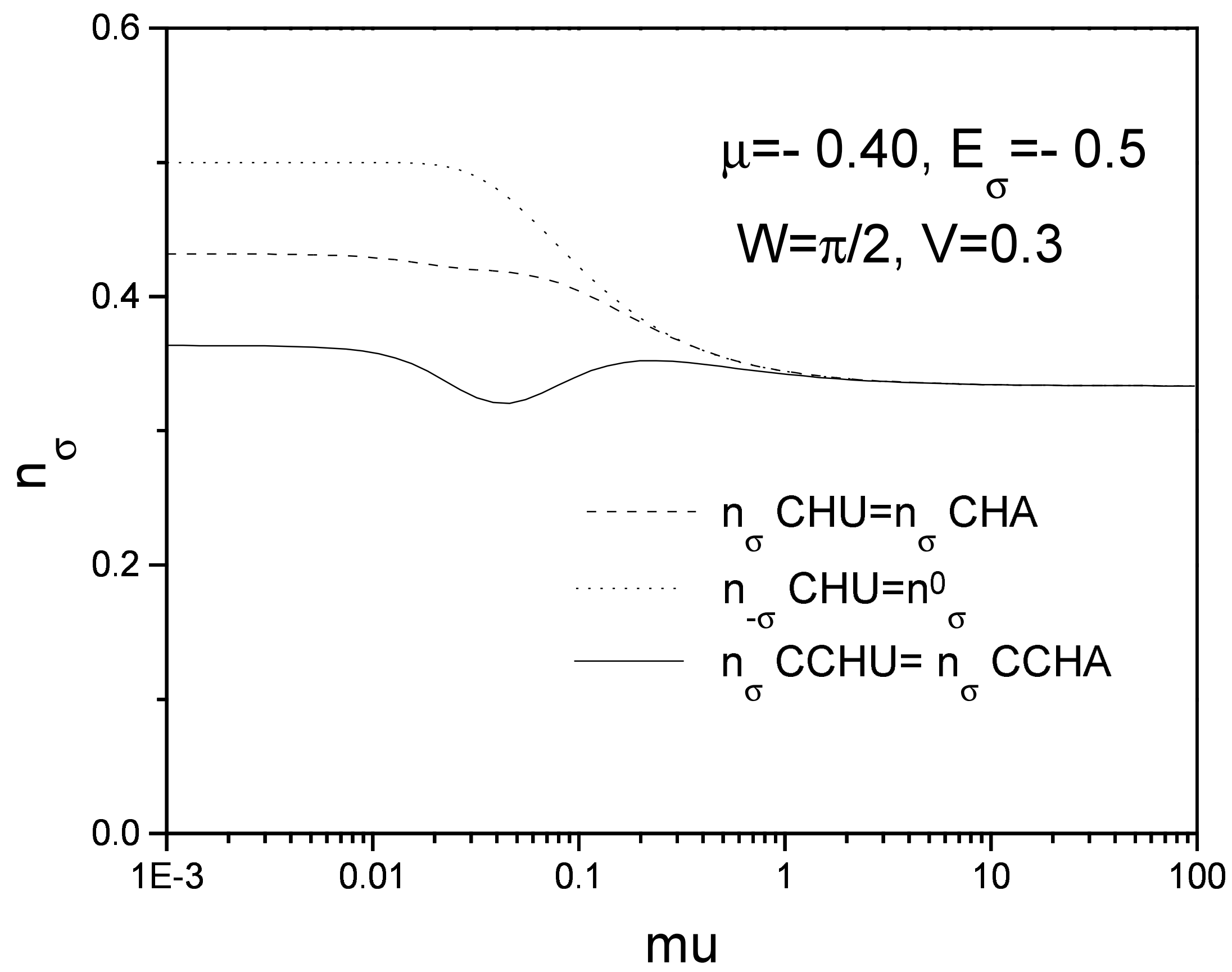




$$
\text { a) } \begin{gathered}
\text { b) } \\
\text { मी }
\end{gathered}
$$




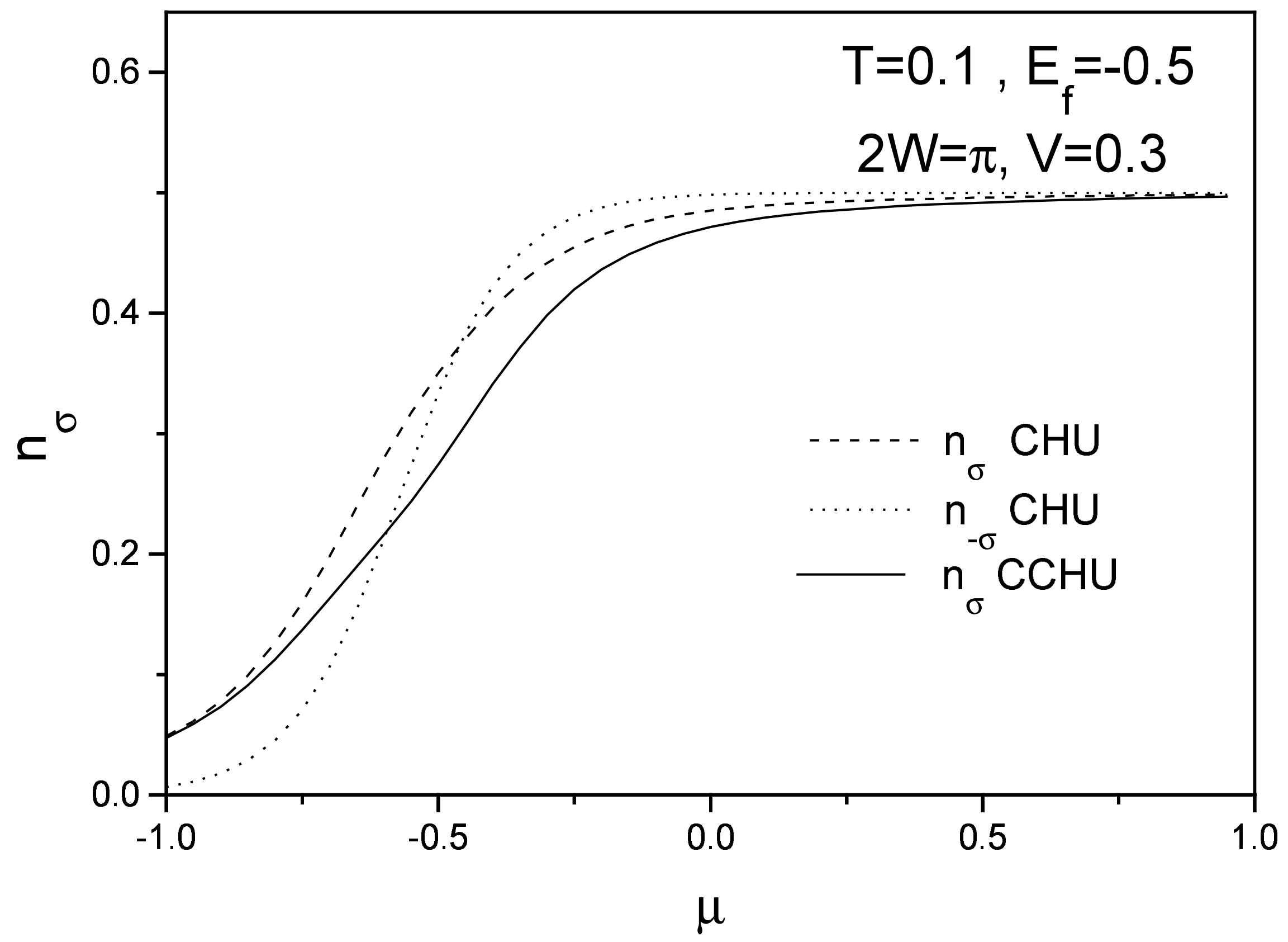

